\title{
Does Particulate Matter Modify the Short-Term Association between Heat Waves and Hospital Admissions for Cardiovascular Diseases in Greater Sydney, Australia?
}

\author{
Marissa Parry ${ }^{1}$, Donna Green ${ }^{1,2,3, * \mathbb{D}}$, Ying Zhang ${ }^{4}$ and Andrew Hayen 5 \\ 1 Climate Change Research Centre, University of New South Wales, Sydney, NSW 2052, Australia \\ 2 ARC Centre of Excellence for Climate Extremes, University of New South Wales, \\ Sydney, NSW 2052, Australia \\ 3 Australia and NHMRC Centre for Air Pollution, Energy and Health Research, University of Sydney, \\ Sydney, NSW 2052, Australia \\ 4 School of Public Health, University of Sydney, Sydney, NSW 2006, Australia \\ 5 Faculty of Health, University of Technology Sydney, Sydney, NSW 2007, Australia \\ * Correspondence: donna.green@unsw.edu.au
}

Received: 20 January 2019; Accepted: 31 July 2019; Published: 5 September 2019

\begin{abstract}
Little is known about the potential interactive effects of heat waves and ambient particulate matter on cardiovascular morbidity. A time-stratified case-crossover design was used to examine whether particulate matter $\left(\mathrm{PM}_{10}\right)$ modifies the association between heat waves and emergency hospital admissions for six cardiovascular diseases in Greater Sydney, Australia during the warm season for 2001-2013. We estimated and compared the effect of heat waves on high- and low-level $\mathrm{PM}_{10}$ days at $\operatorname{lag}_{0}-\mathrm{lag}_{2}$, adjusting for dew-point temperature, ambient ozone, ambient nitrogen dioxide, and public holidays. We also investigated the susceptibility of both younger (0-64 years) and older populations (65 years and above), and tested the sensitivity of three heat wave definitions. Stronger heat wave effects were observed on high- compared to low-level $\mathrm{PM}_{10}$ days for emergency hospital admissions for cardiac arrest for all ages combined, 0-64 years and 65 years and above; conduction disorders for 0-64 years; and hypertensive diseases for all ages combined and 0-64 years. Overall, we found some evidence to suggest that $\mathrm{PM}_{10}$ may modify the association between heat waves and hospital admissions for certain cardiovascular diseases, although our findings largely differed across disease, age group, lag, and heat wave definition.
\end{abstract}

Keywords: heat waves; particulate matter; Sydney; Australia; hospital admissions; cardiovascular disease

\section{Introduction}

Cardiovascular disease is a major cause of death both worldwide and in Australia [1,2]. Some studies have shown that high temperatures and heat waves are associated with increased risk of hospitalisation for cardiovascular diseases generally [3-5], and specific cardiovascular diseases including ischemic heart disease and cardiac (or heart) dysrhythmias $[3,4,6]$. Elevated temperature and heat waves have also been shown to be associated with an increased risk of out-of-hospital cardiac arrest [7]. A short lag effect has been observed, with positive associations between high temperatures and hospitalisations for cardiovascular diseases reported on the same day of exposure [4] and between 1-3 days after exposure [3]. Other studies, however, including two meta-analyses, have reported null or negative associations between high temperatures and hospital admissions for cardiovascular diseases [8-11], but Phung et al. [10] reported a small, positive heat wave effect. 
Ambient particulate matter with an aerodynamic diameter less than $10 \mu \mathrm{m}$, known as particulate matter $\left(\mathrm{PM}_{10}\right)$, is comprised of both solid particles and liquid droplets from natural and anthropogenic sources [12]. Levels and mixtures of $\mathrm{PM}_{10}$ can depend on season and temperature, with bushfire smoke and dust storms being important sources during the warm season in Australia, and wood heaters an important source in the cool season [13]. Studies have shown that elevated levels of $\mathrm{PM}_{10}$ are associated with an increased risk of hospitalisation for all cardiovascular or cardiac diseases [14-16] and specific diseases including ischemic heart disease [17,18], heart failure [19], and heart arrhythmias and conduction disorders [15], particularly among the elderly. Elevated levels of $\mathrm{PM}_{10}$ have also been shown to be associated with an increased risk of out-of-hospital cardiac arrest [20]. A few studies have assessed, or controlled for, the potential confounding effects of $\mathrm{PM}_{10}$ when estimating the association between extreme heat and hospitalisations for cardiovascular diseases (e.g., [21,22]).

Little is known about the potential joint or interactive effects of high temperatures, particularly heat waves, and $\mathrm{PM}_{10}$ on cardiovascular health outcomes. This is concerning given that the joint effect of weather and air pollution on health outcomes is thought to be greater than the risk derived from the individual impacts of these two exposures [23]. There is also some suggestion that an interactive effect between air pollution and temperature may be biologically plausible [24]. Some studies from Europe and Asia have investigated whether temperature modifies the association between $\mathrm{PM}_{10}$ and all-cause and/or cardiovascular mortality [25-30]. Most of these studies have generally found stronger associations at high compared to moderate or low level temperatures, although such evidence of effect modification has not been consistently statistically significant. However, Cheng and Kan [28] found a statistically significant interaction between low, but not high, temperature and $\mathrm{PM}_{10}$ on total and cardiovascular mortality in Shanghai, China.

Few studies have investigated whether $\mathrm{PM}_{10}$ modifies the association between high temperatures, particularly heat waves, and cardiovascular health outcomes. Some have found stronger associations between high temperatures or heat waves and all-cause and/or cardiovascular mortality at higher levels of $\mathrm{PM}_{10}$, although not all have reported evidence of statistical significance [30-33]. Other studies have found no evidence of an interaction between temperature and $\mathrm{PM}_{10}$ on mortality [34,35]. Little work, however, has examined whether $\mathrm{PM}_{10}$ modifies the association between temperature or heat waves and cardiovascular morbidity, particularly cause-specific cardiovascular morbidity. One Australian study found that $\mathrm{PM}_{10}$ modified the association between temperature and cardiovascular hospital admissions at different lags in Brisbane, however it found little evidence of effect modification for cardiovascular emergency presentations [36]. Further, a recent Korean study found no evidence of a significant interactive effect between heat waves and $\mathrm{PM}_{10}$ on out-of-hospital cardiac arrest [7].

The frequency, intensity, and duration of heat waves is expected to increase in the future under climate change across most land areas globally, including Australia [37,38]. It is therefore important to clarify and enhance our understanding of the association between heat waves and cardiovascular morbidity to inform climate change adaptation planning in the health sector. This study aimed to examine whether $\mathrm{PM}_{10}$ modifies the short-term association between heat waves and hospital admissions for specific cardiovascular diseases in Greater Sydney, Australia. We investigated the susceptibility of both younger (0-64 years) and older populations (65 years and above), and tested the sensitivity of three heat wave definitions.

\section{Materials and Methods}

\subsection{Meteorological Data}

Daily weather data for all stations located in the Sydney Statistical Division (SSD) with near complete coverage of the period of 2001 to 2013 were obtained from the Australian Government's Bureau of Meteorology $(n=17)$. Before identifying extreme temperature events in a climate time series, such as summer heat waves, it is important that the data undergo quality control checks [39]. This is because it is possible for incorrect data entries to be considered as real "extreme" values and 
included in further analyses [39]. To ensure our observational weather data was of the highest possible quality, we performed a series of quality control checks on the observed daily maximum, minimum, and dew-point temperature values for each weather station, and also tested for inhomogeneities in each daily maximum and minimum time series to inspect their overall quality. High quality stations $(n=15)$ were then used to calculate the respective city-wide averages for each temperature metric if they had a total missing value count of $\leq 2.5 \%$ of the study period. The missing value threshold was set at $\leq 2.5 \%$ to maximise the number of stations included in the calculation of the average and subsequent spatial coverage of the SSD, while also ensuring that the quality of those stations that were included remained high. The daily average mean temperature was calculated as the mean of the city-wide daily average maximum and minimum temperature values. For dew-point temperature, as the observations were recorded at 3-hour intervals over a 24-hour period, the city-wide average value for each time interval was first calculated with those stations where the missing value count was $\leq 2.5 \%$ of the study period, then the overall 24 -hour daily average was calculated from these averaged time interval values.

In the absence of a standard heat wave definition, we selected and compared three heat wave definitions for this study. Previous studies have shown that the choice of heat wave definition can alter the magnitude and statistical significance of the association between heat waves and adverse health outcomes $[40,41]$. We defined a heat wave as two or more consecutive days where the temperature metric (three temperature metrics were selected and compared: maximum temperature (HWD01), mean temperature (HWD02), and minimum temperature (HWD03)) is greater than or equal to the 90th percentile of the warm season (1 November to 31 March) during 2001 to 2013. We compared heat wave definitions with alternative temperature metrics, rather than temperature thresholds or durations, to ensure that we kept an adequate number of heat wave days to conduct the analysis.

\subsection{Ambient Air Pollution Data}

Daily ambient air pollution data for all stations located in the SSD were obtained from the NSW Office of Environment and Heritage for 2001 to 2013. Daily data were obtained for the following air pollutants and used in this study: ozone ( $1 \mathrm{~h}$ average $24 \mathrm{~h}$ maximum value (pphm)); nitrogen dioxide ( $1 \mathrm{~h}$ average $24 \mathrm{~h}$ maximum value $(\mathrm{pphm})$ ), and particulate matter (particles with an aerodynamic diameter of less than $10 \mu \mathrm{m}, \mathrm{PM}_{10}$ ) ( $1 \mathrm{~h}$ average $24 \mathrm{~h}$ average value). The NSW Office of Environment and Heritage follows several quality assurance procedures to ensure the data are precise, accurate, representative, and comparable [42]. Negative daily values were assigned a value of 0 . Stations that had a missing value count of $\leq 5 \%$ of the study period were used to calculate the daily city-wide average for each pollutant. Junger and Ponce de Leon [43] regarded a missing data level of $5 \%$ as the best-case scenario in their application of time-series air pollution data. Similar to the threshold selection for our meteorological data, a threshold of $5 \%$ was optimal in allowing us to maximise the number of stations included in the calculation of the average and subsequent spatial coverage of the $\mathrm{SSD}$, while also ensuring that the quality of those stations that were included remained high. $\mathrm{PM}_{2.5}$ (ambient particulate matter with an aerodynamic diameter less than $2.5 \mu \mathrm{m}$ ) was not considered in this study given the smaller spatial and temporal coverage of the data across the Greater Sydney region.

\subsection{Health Data}

Individual-level daily hospital admission records with a principal diagnosis of I00-I99 (ICD-10-AM) for all public and private hospitals located in the SSD were obtained from the NSW Ministry of Health, Admitted Patient Data Collection, for 2001 to 2013 as part of a larger dataset $(n=1,570,805)$. All exact duplicate records were extracted and removed $(n=1,570,741,64$ records removed), as well those records with an admission date outside of 1 July 2001-30 June 2013 ( $n=1,499,661,71,080$ records removed). Records that were classified as "emergency" hospital admissions (EHAs) were then selected for analysis to eliminate "pre-planned" hospital admissions ( $n=1,132,737$, records removed 366924) [44]. We then extracted and removed remaining records with an implausible, unknown, or missing entry for age (ranged deemed plausible: $0-115$ years) or sex (required entry: male or female) $(n=1,132,705$, records 
removed 32). Those records with a principal diagnosis of ischemic heart disease (ICD-10-AM: I20-I25), heart failure (ICD-10-AM: I50), cardiac arrest (ICD-10-AM: I46), heart arrhythmia (ICD-10-AM: 147-I49), conduction disorders (ICD-10-AM: I44-I45), and hypertensive diseases (ICD-10-AM: I10-I15) were then selected and aggregated into daily counts. To investigate the susceptibility of both younger and older populations, we stratified the data into two age groups: 0-64 years and 65 years and above.

\subsection{Study Design and Statistical Analysis}

We used a time-stratified case-crossover study design $[45,46]$. This design has been used in previous studies to estimate the association between heat waves and hospital admissions $[47,48]$, and has been shown to produce similar results to the alternate time-series design [49]. The design is equivalent to a matched pair case-control design: it compares a case's exposure on the day of an adverse health event (e.g., hospital admission) to their exposure on control days (or referent times), which are selected before and/or after the event $[46,50,51]$. Since each case acts as their own control, personal characteristics such as sex and smoking status are controlled for by matching [51]. We used the time-stratified approach to select control days to avoid potential bias introduced by other approaches, such as the unidirectional and bidirectional designs [46]. We matched cases and controls on day of the week and within the same month, and thus controlled for the confounding effects of season and long-term trends by design.

We used conditional logistic regression to estimate the association between heat waves and EHAs for our six selected cardiovascular diseases. We first estimated the association with and without adjusting for daily average $\mathrm{PM}_{10}$ at $\operatorname{lag}_{0}$. All of the models included daily average dew-point temperature, daily average nitrogen dioxide, daily average ozone, and public holidays as covariates. More specifically, we adjusted for daily average dew-point temperature [52] using a natural cubic spline $(\mathrm{df}=3$, knots at quantiles), daily average nitrogen dioxide ( $1 \mathrm{~h}$ average $24 \mathrm{~h}$ maximum value $(\mathrm{pphm})$ ), daily average ozone ( $1 \mathrm{~h}$ maximum $24 \mathrm{~h}$ average value $(\mathrm{pphm}))$, and public holidays. To determine the most appropriate way to model dew-point temperature, we conducted sensitivity tests modelling this variable as a natural cubic spline with 3 and 2 degrees of freedom, and as a linear variable at lag. As the coefficients of the heat wave effect were largely similar across the three modelling approaches, we selected to model dew-point temperature as a natural cubic spline with 3 degrees of freedom to be consistent with previous work [53].

To examine whether $\mathrm{PM}_{10}$ modifies the association between heat waves and EHAs for our six selected cardiovascular diseases, we estimated and compared heat wave effects on days with high and low levels of $\mathrm{PM}_{10}$ at $\operatorname{lag}_{0}-\mathrm{lag}_{2}$. High and low level $\mathrm{PM}_{10}$ days were defined as those where the daily average $\mathrm{PM}_{10}$ value was $\geq 90$ th and $<90$ th percentile of the warm season during 2001 to 2013, respectively (Note: 90th percentile of the distribution was equal to $30.52 \mu \mathrm{g} / \mathrm{m}^{3}$ ). We created an interaction term between high and low level $\mathrm{PM}_{10}$ days $(1=$ high, $0=$ low $)$ and heat wave days $(1=$ yes, $0=$ no). This term was added to the model, along with the respective individual variables and potential confounding variables described in the previous paragraph. We selected the threshold of the 90th percentile for two main reasons: to ensure there was a reasonably equal distribution of high and low level $\mathrm{PM}_{10}$ days across heat wave days for the three definitions for a fair comparison and to compare and estimate heat wave effects on days with the more extreme values of $\mathrm{PM}_{10}$.

The statistical analyses were conducted in the " $R$ " Statistical Computing Environment (Version 3.2.1) using the "season" and "dlnm" packages. As we wanted to examine the impact of summer heat waves, we restricted our analyses to the warm season (1 November to 31 March) for 2001 to 2013. The effects are presented as odds ratio with their corresponding 95\% confidence intervals. The figure is presented on the log scale. A $p$-value of $<0.05$ was considered significant.

This project was approved by the University of New South Wales Human Research Low Risk Ethics Advisory Committee Panel H. 


\section{Results}

Descriptive statistics for selected weather and ambient air pollution variables during the study period are presented in Table 1 . The mean daily average maximum temperature was $26.0{ }^{\circ} \mathrm{C}$, and the mean daily average value of $\mathrm{PM}_{10}$ was $20.43 \mu \mathrm{g} / \mathrm{m}^{3}$.

Table 1. Descriptive statistics for environmental variables in the SSD during the warm season, 2001 to 2013.

\begin{tabular}{lccc}
\hline Environmental Variables & Mean $(\mathrm{SD})$ Value & Maximum Value & Minimum Value \\
\hline Weather (Degrees Celsius $\left.\left({ }^{\circ} \mathrm{C}\right)\right)$ & & & \\
Daily average maximum temperature & $26.40(4.38)$ & 43.99 & 14.41 \\
Daily average mean temperature & $21.34(3.05)$ & 32.38 & 12.47 \\
Daily average minimum temperature & $16.27(2.70)$ & 24.39 & 6.82 \\
Daily average dew-point temperature & $14.92(3.34)$ & 22.10 & -0.13 \\
Ambient Air pollution & & & \\
Daily average ozone $(\mathrm{pphm})$ & $3.78(1.48)$ & 11.52 & 1.04 \\
Daily average $\mathrm{PM}_{10}\left(\mu \mathrm{gg} / \mathrm{m}^{3}\right)$ & $20.43(11.48)$ & 222.30 & 4.57 \\
Daily average nitrogen dioxide $(\mathrm{pphm})$ & $1.44(0.59)$ & 4.56 & 0.29 \\
\hline
\end{tabular}

Table 2 shows descriptive statistics for selected EHAs for six cardiovascular diseases for all ages combined and two age groups: 0-64 years and 65 years and over. Ischemic heart disease had the highest number of total EHAs during the study period with 68,334, while cardiac arrest had the lowest with 1861. For each cardiovascular disease, the older age group had a higher number of EHAs than the younger age group.

Table 2. Descriptive statistics for EHAs for six cardiovascular diseases in the Sydney Statistical Division (SSD) during the warm season, 2001 to 2013.

\begin{tabular}{|c|c|c|c|c|c|}
\hline & $\begin{array}{l}\text { ICD Code } \\
\text { (ICD-10-AM) }\end{array}$ & $\begin{array}{l}\text { Total } \\
\text { Count }\end{array}$ & $\begin{array}{c}\text { Median (IQR) } \\
\text { Daily Value }\end{array}$ & $\begin{array}{l}\text { Maximum } \\
\text { Daily Value }\end{array}$ & $\begin{array}{c}\text { Minimum } \\
\text { Daily Value }\end{array}$ \\
\hline \multicolumn{6}{|l|}{ Cardiovascular Disease } \\
\hline Ischemic Heart Disease & I20-I25 & & & & \\
\hline All ages & & 68,334 & $37(31-43)$ & 70 & 14 \\
\hline $0-64$ years & & 28,497 & $15(12-19)$ & 34 & 3 \\
\hline 65 years and over & & 39,837 & $22(18-26)$ & 46 & 5 \\
\hline Heart Failure & $\mathrm{I} 50$ & & & & \\
\hline All ages & & 24,721 & $13(10-17)$ & 31 & 2 \\
\hline $0-64$ years & & 3470 & $2(1-3)$ & 9 & 0 \\
\hline 65 years and over & & 21,251 & $11(9-14)$ & 28 & 0 \\
\hline Cardiac Arrest & $\mathrm{I} 46$ & & & & \\
\hline All ages & & 1861 & $1(0-2)$ & 6 & 0 \\
\hline $0-64$ years & & 802 & $0(0-1)$ & 4 & 0 \\
\hline 65 years and over & & 1059 & $0(0-1)$ & 4 & 0 \\
\hline Heart Arrhythmia & I47-I49 & & & & \\
\hline All ages & & 32,682 & $18(14-21)$ & 36 & 5 \\
\hline $0-64$ years & & 12,461 & $7(5-9)$ & 19 & 0 \\
\hline 65 years and over & & 20,221 & $11(8-14)$ & 25 & 1 \\
\hline Conduction Disorders & I44-I45 & & & & \\
\hline All ages & & 3070 & $1(1-3)$ & 7 & 0 \\
\hline $0-64$ years & & 641 & $0(0-1)$ & 4 & 0 \\
\hline 65 years and over & & 2429 & $1(0-2)$ & 7 & 0 \\
\hline Hypertensive Diseases & I10-I15 & & & & \\
\hline All ages & & 3859 & $2(1-3)$ & 9 & 0 \\
\hline $0-64$ years & & 1571 & $1(0-1)$ & 6 & 0 \\
\hline 65 years and over & & 2288 & $1(0-2)$ & 7 & 0 \\
\hline
\end{tabular}


A summary of the heat wave characteristics for each heat wave definition used is provided in Table 3. HWD03 had the highest total number of heat wave days during the study period and the longest average heat wave duration of 2.92 days. HWD02 had the highest number of total heat wave events with 43 .

Table 3. Summary of heat wave characteristics for each heat wave definition used.

\begin{tabular}{ccccc}
\hline $\begin{array}{c}\text { Heat Wave } \\
\text { Definition }\end{array}$ & $\begin{array}{c}\text { Total Number of } \\
\text { Heat Wave Days }\end{array}$ & $\begin{array}{c}\text { Total Number of } \\
\text { Heat Wave Events }\end{array}$ & $\begin{array}{c}\text { Average Intensity }{ }^{\text {a of }} \\
\text { Heat Wave Day }\left({ }^{\circ} \mathbf{C}\right)\end{array}$ & $\begin{array}{c}\text { Average Duration of } \\
\text { Heat Wave (in Days) }\end{array}$ \\
\hline HWD01 & 98 & 38 & 35.19 & 2.58 \\
HWD02 & 113 & 43 & 27.31 & 2.63 \\
HWD03 & 114 & 39 & 20.75 & 2.92 \\
\hline
\end{tabular}

${ }^{a}$ The average intensity was calculated using the temperature metric used in each heat wave definition.

Figure 1 shows the association between heat wave days and EHAs for six cardiovascular diseases with and without controlling for daily average $\mathrm{PM}_{10}$ at $\operatorname{lag}_{0}$ for all ages. For all diseases and across the three heat wave definitions, controlling for daily average $\mathrm{PM}_{10}$ had little effect on the health risk estimates. Negative associations were found between heat wave days and EHAs for heart arrhythmia and hypertensive diseases for all three heat wave definitions, although these associations were not statistically significant. Negative associations were also found between heat wave days and EHAs for ischemic heart disease, heart failure, and conduction disorders for HWD01 and HWD02, and small positive associations were found for HWD03. The negative associations found for EHAs for ischemic heart disease for HWD01 and HWD02 were statistically significant. Small, positive associations were found between heat wave days and EHAs for cardiac arrest for HWD01 and HWD02, and negative associations were found for HWD03.

Table 4 shows the association between heat wave days and EHAs for six cardiovascular diseases at two levels of $\mathrm{PM}_{10}$ (high: $\geq 90$ th percentile; and low: <90th percentile) for all ages at lag 0 and $\operatorname{lag}_{1}$. The results for $\operatorname{lag}_{2}$ are presented in Table A1 in Appendix A. A positive, statistically significant interaction was found between heat wave and high-level $\mathrm{PM}_{10}$ days on EHAs for hypertensive diseases at $\operatorname{lag}_{1}$ for HWD03, meaning that there was a stronger effect on EHAS on high-level PM $_{10}$ days than on low-level $\mathrm{PM}_{10}$ days. Heat wave effects were also stronger on high-level $\mathrm{PM}_{10}$ days for hypertensive diseases for HWD03 at $\operatorname{lag}_{0}$ and $\operatorname{lag}_{2}$, but the $p$-value of the interaction term was not statistically significant. The impact of heat waves on EHAs for cardiac arrest was generally found to be stronger on days with high levels of $\mathrm{PM}_{10}$ across most lags and definitions, although none of the interaction terms were statistically significant. A negative, statistically significant interaction was found between heat wave and high-level $\mathrm{PM}_{10}$ days on EHAs for ischemic heart disease at $\operatorname{lag}_{2}$ for HWD01 (meaning that there was a weaker effect on EHAS on high-level $\mathrm{PM}_{10}$ days than on low-level $\mathrm{PM}_{10}$ days), but not at $\operatorname{lag}_{0}$ or $\operatorname{lag}_{1}$.

Table 5 shows the association between heat wave days and EHAs for six cardiovascular diseases at two levels of $\mathrm{PM}_{10}$ (high: $\geq 90$ th percentile; and low: <90th percentile) for younger and older populations at $\operatorname{lag}_{0}$ and $\operatorname{lag}_{1}$. The results for $\operatorname{lag}_{2}$ are presented in Table A2 in the Appendix A. A positive, statistically significant interaction was found between heat wave and high-level $\mathrm{PM}_{10}$ days on EHAs for cardiac arrest in the older age group for HWD01 at lag and $\mathrm{lag}_{2}$, and for HWD02 at lag ${ }_{1}$. Heat wave effects were also found to be stronger on high-level $\mathrm{PM}_{10}$ days at $\operatorname{lag}_{0}$ for HWD02, and at $\operatorname{lag}_{0}$ and $\operatorname{lag}_{1}$ for HWD03 in the younger age group, but no evidence of a statistically significant interaction was found. The impact of heat waves on EHAs for conduction disorders was stronger on high-level $\mathrm{PM}_{10}$ days for all definitions and lags, and on EHAs for hypertensive diseases for HWD02 and HWD03 at all lags and $\operatorname{lag}_{1}$ for HWD01 in the younger population. Stronger heat wave effects on high- compared to low-level $\mathrm{PM}_{10}$ days were found for EHAs for heart failure at lag 1 for HWD03 in the older age group. A negative, statistically significant interaction was found between heat wave and high-level $\mathrm{PM}_{10}$ days on EHAs for heart arrhythmia for HWD01 at lag 1 in the younger age group. 


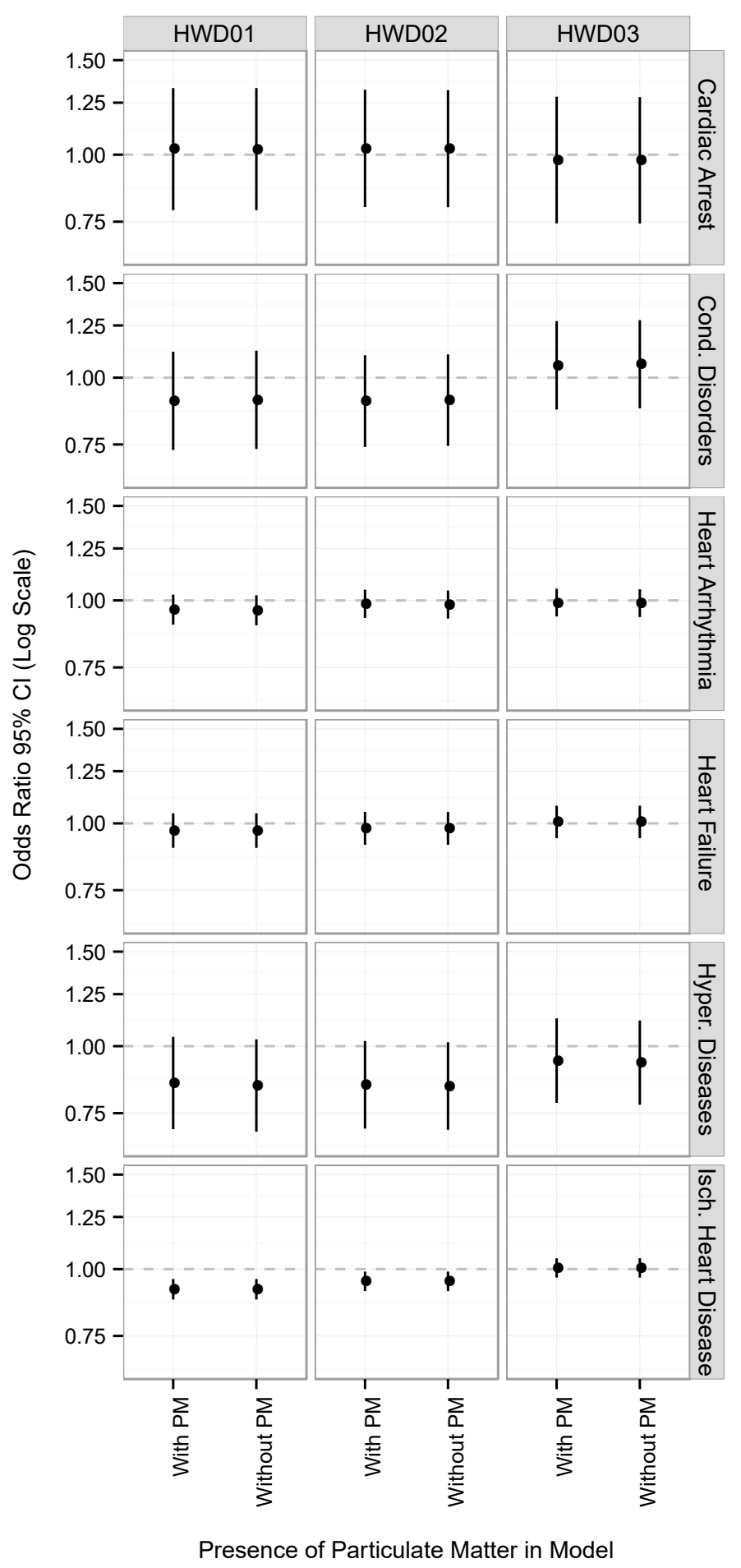

Figure 1. The association between heat wave days and "emergency" hospital admissions (EHAs) for six cardiovascular diseases with and without controlling for daily average particulate matter $\left(\mathrm{PM}_{10}\right)$ at $\operatorname{lag}_{0}$ in the SSD during the warm season, 2001 to 2013. Note: Cond. Disorders is conduction disorders; Hyper. Diseases is hypertensive diseases; Isch. Heart Disease is Ischemic Heart Disease. 
Table 4. The effect of heat wave days on EHAs for six cardiovascular diseases on days with high levels of PM 10 compared to days with low levels of PM 10 in the SSD during the warm season, 2001 to 2013, for all ages. Effects are presented as odds ratios with their corresponding $95 \%$ confidence intervals.

\begin{tabular}{|c|c|c|c|c|c|c|c|c|c|c|c|c|}
\hline & \multicolumn{4}{|c|}{ HWD01 } & \multicolumn{4}{|c|}{ HWD02 } & \multicolumn{4}{|c|}{ HWD03 } \\
\hline & \multicolumn{2}{|c|}{$\operatorname{Lag}_{0}$} & \multicolumn{2}{|c|}{$\operatorname{Lag}_{1}$} & \multicolumn{2}{|c|}{$\mathrm{Lag}_{0}$} & \multicolumn{2}{|c|}{$\operatorname{Lag}_{1}$} & \multicolumn{2}{|c|}{$\operatorname{Lag}_{0}$} & \multicolumn{2}{|c|}{$\operatorname{Lag}_{1}$} \\
\hline & $\begin{array}{c}\text { Heat Effect } \\
\text { Low } \mathrm{PM}_{10}\end{array}$ & $\begin{array}{l}\text { Heat Effect } \\
\text { High } \text { PM }_{10}\end{array}$ & $\begin{array}{r}\text { Heat Effect } \\
\text { Low } \text { PM }_{10}\end{array}$ & $\begin{array}{l}\text { Heat Effect } \\
\text { High } \text { PM }_{10}\end{array}$ & $\begin{array}{r}\text { Heat Effect } \\
\text { Low } \text { PM }_{10}\end{array}$ & $\begin{array}{l}\text { Heat Effect } \\
\text { High } \text { PM }_{10}\end{array}$ & $\begin{array}{r}\text { Heat Effect } \\
\text { Low } \mathbf{P M}_{10}\end{array}$ & $\begin{array}{l}\text { Heat Effect } \\
\text { High } \text { PM }_{10}\end{array}$ & $\begin{array}{r}\text { Heat Effect } \\
\text { Low } \mathbf{P M}_{10}\end{array}$ & $\begin{array}{l}\text { Heat Effect } \\
\text { High } \text { PM }_{10}\end{array}$ & $\begin{array}{r}\text { Heat Effect } \\
\text { Low } \mathbf{P M}_{10}\end{array}$ & $\begin{array}{l}\text { Heat Effect } \\
\text { High } \mathbf{P M}_{10}\end{array}$ \\
\hline Cardiovascular Disease & & & & & & & & & & & & \\
\hline Ischemic Heart & 0.92 & 0.92 & 0.97 & 0.94 & 0.98 & 0.92 & 0.98 & 0.92 & 1.03 & 0.95 & 1.04 & 1.01 \\
\hline Disease & $(0.87,0.98)$ & $(0.86,0.98)$ & $(0.92,1.02)$ & $(0.87,1.01)$ & $(0.93,1.03)$ & $(0.86,0.97)$ & $(0.94,1.03)$ & $(0.85,0.99)$ & $(0.98,1.07)$ & $(0.87,1.03)$ & $(1.00,1.08)$ & $(0.93,1.09)$ \\
\hline Heart Failure & $\begin{array}{c}0.94 \\
(0.85,1.04)\end{array}$ & $\begin{array}{c}1.00 \\
(0.90,1.11)\end{array}$ & $\begin{array}{c}0.83 \\
(0.76,0.90)\end{array}$ & $\begin{array}{c}0.94 \\
(0.83,1.06)\end{array}$ & $\begin{array}{c}0.99 \\
(0.90,1.08)\end{array}$ & $\begin{array}{c}0.97 \\
(0.87,1.08)\end{array}$ & $\begin{array}{c}0.89 \\
(0.83,0.95)\end{array}$ & $\begin{array}{c}0.94 \\
(0.83,1.06)\end{array}$ & $\begin{array}{c}1.01 \\
(0.93,1.09)\end{array}$ & $\begin{array}{c}1.01 \\
(0.88,1.16)\end{array}$ & $\begin{array}{c}0.95 \\
(0.89,1.01)\end{array}$ & $\begin{array}{c}0.93 \\
(0.80,1.07)\end{array}$ \\
\hline Cardiac Arrest & $\begin{array}{c}1.06 \\
(0.73,1.55)\end{array}$ & $\begin{array}{c}0.99 \\
(0.69,1.41)\end{array}$ & $\begin{array}{c}1.05 \\
(0.77,1.41)\end{array}$ & $\begin{array}{c}1.30 \\
(0.86,1.97)\end{array}$ & $\begin{array}{c}0.88 \\
(0.62,1.23)\end{array}$ & $\begin{array}{c}1.22 \\
(0.86,1.74)\end{array}$ & $\begin{array}{c}1.08 \\
(0.84,1.40)\end{array}$ & $\begin{array}{c}1.41 \\
(0.93,2.14)\end{array}$ & $\begin{array}{c}0.93 \\
(0.68,1.26)\end{array}$ & $\begin{array}{c}1.13 \\
(0.70,1.83)\end{array}$ & $\begin{array}{c}1.21 \\
(0.95,1.55)\end{array}$ & $\begin{array}{c}1.24 \\
(0.75,2.07)\end{array}$ \\
\hline Heart Arrhythmia & $\begin{array}{c}0.95 \\
(0.86,1.04)\end{array}$ & $\begin{array}{c}1.01 \\
(0.92,1.10)\end{array}$ & $\begin{array}{c}0.99 \\
(0.92,1.06)\end{array}$ & $\begin{array}{c}0.94 \\
(0.84,1.04)\end{array}$ & $\begin{array}{c}0.98 \\
(0.92,1.06)\end{array}$ & $\begin{array}{c}1.02 \\
(0.93,1.12)\end{array}$ & $\begin{array}{c}0.99 \\
(0.93,1.05)\end{array}$ & $\begin{array}{c}0.97 \\
(0.87,1.08)\end{array}$ & $\begin{array}{c}1.00 \\
(0.94,1.07)\end{array}$ & $\begin{array}{c}0.96 \\
(0.85,1.08)\end{array}$ & $\begin{array}{c}1.06 \\
(1.00,1.12)\end{array}$ & $\begin{array}{c}0.96 \\
(0.85,1.09)\end{array}$ \\
\hline Conduction & 0.84 & 1.04 & 0.91 & 0.92 & 0.90 & 0.96 & 0.94 & 0.87 & 1.11 & 0.89 & 0.89 & 0.86 \\
\hline Disorders & $(0.63,1.12)$ & $(0.77,1.41)$ & $(0.73,1.13)$ & $(0.64,1.33)$ & $(0.71,1.14)$ & $(0.71,1.30)$ & $(0.77,1.13)$ & $(0.60,1.25)$ & $(0.91,1.37)$ & $(0.60,1.31)$ & $(0.74,1.07)$ & $(0.57,1.30)$ \\
\hline Hypertensive & 0.86 & 0.87 & 0.91 & 0.92 & 0.88 & 0.82 & 0.91 & 0.91 & 0.90 & 1.10 & 0.86 & $1.30 *$ \\
\hline Diseases & $(0.65,1.13)$ & $(0.66,1.16)$ & $(0.83,1.25)$ & $(0.65,1.30)$ & $(0.70,1.12)$ & $(0.61,1.09)$ & $(0.76,1.09)$ & $(0.64,1.29)$ & $(0.74,1.10)$ & $(0.75,1.60)$ & $(0.73,1.02)$ & $(0.90,1.89)$ \\
\hline
\end{tabular}

* Denotes a statistically significant positive interaction term $p$-value $(<0.05)$ 
Table 5. The effect of heat wave days on EHAs for six cardiovascular diseases on days with high levels of $\mathrm{PM}_{10}$ compared to days with low levels of $\mathrm{PM}_{10}$ in the SSD during the warm season, 2001 to 2013, for those aged 0-64 years and 65 years and over. Effects are presented as odds ratios with their corresponding 95\% confidence intervals.

\begin{tabular}{|c|c|c|c|c|c|c|c|c|c|c|c|c|}
\hline & \multicolumn{4}{|c|}{ HWD01 } & \multicolumn{4}{|c|}{ HWD02 } & \multicolumn{4}{|c|}{ HWD03 } \\
\hline & \multicolumn{2}{|c|}{$\mathrm{Lag}_{0}$} & \multicolumn{2}{|c|}{$\operatorname{Lag}_{1}$} & \multicolumn{2}{|c|}{$\operatorname{Lag}_{0}$} & \multicolumn{2}{|c|}{$\operatorname{Lag}_{1}$} & \multicolumn{2}{|c|}{$\operatorname{Lag}_{0}$} & \multicolumn{2}{|c|}{$\operatorname{Lag}_{1}$} \\
\hline & $\begin{array}{r}\text { Heat Effect } \\
\text { Low } \mathrm{PM}_{10}\end{array}$ & $\begin{array}{l}\text { Heat Effect } \\
\text { High } \text { PM }_{10}\end{array}$ & $\begin{array}{l}\text { Heat Effect } \\
\text { Low } \mathrm{PM}_{10}\end{array}$ & $\begin{array}{l}\text { Heat Effect } \\
\text { High } \text { PM }_{10}\end{array}$ & $\begin{array}{l}\text { Heat Effect } \\
\text { Low } \mathrm{PM}_{10}\end{array}$ & $\begin{array}{l}\text { Heat Effect } \\
\text { High } \text { PM }_{10}\end{array}$ & $\begin{array}{l}\text { Heat Effect } \\
\text { Low } \mathrm{PM}_{10}\end{array}$ & $\begin{array}{l}\text { Heat Effect } \\
\text { High } \text { PM }_{10}\end{array}$ & $\begin{array}{l}\text { Heat Effect } \\
\text { Low } \mathrm{PM}_{10}\end{array}$ & $\begin{array}{l}\text { Heat Effect } \\
\text { High } \mathbf{P M}_{10}\end{array}$ & $\begin{array}{r}\text { Heat Effect } \\
\text { Low } \mathrm{PM}_{10}\end{array}$ & $\begin{array}{l}\text { Heat Effect } \\
\text { High PM } \text { PM }_{10}\end{array}$ \\
\hline \multicolumn{13}{|c|}{$\begin{array}{l}\text { Cardiovascular Disease } \\
\text { Ischemic Heart Disease }\end{array}$} \\
\hline $\begin{array}{l}65 \text { years and over } \\
\text { Heart Failure }\end{array}$ & $\begin{array}{c}0.97 \\
(0.88,1.07) \\
0.89 \\
(0.82,0.96)\end{array}$ & $\begin{array}{c}0.94 \\
(0.85,1.03) \\
0.90 \\
(0.83,0.98)\end{array}$ & $\begin{array}{c}1.03 \\
(0.96,1.11) \\
0.93 \\
(0.87,0.99)\end{array}$ & $\begin{array}{c}1.00 \\
(0.89,1.11) \\
0.89 \\
(0.81,0.98)\end{array}$ & $\begin{array}{c}1.02 \\
(0.94,1.11) \\
0.95 \\
(0.88,1.01)\end{array}$ & $\begin{array}{c}0.92 \\
(0.84,1.02) \\
0.91 \\
(0.84,0.99)\end{array}$ & $\begin{array}{c}1.00 \\
(0.94,1.07) \\
0.97 \\
(0.92,1.03)\end{array}$ & $\begin{array}{c}0.94 \\
(0.84,1.05) \\
0.90 \\
(0.82,0.99)\end{array}$ & $\begin{array}{c}1.07 \\
(1.00,1.15) \\
0.99 \\
(0.93,1.05)\end{array}$ & $\begin{array}{c}1.01 \\
(0.89,1.14) \\
0.90 \\
(0.81,1.01)\end{array}$ & $\begin{array}{c}1.08 \\
(1.02,1.15) \\
1.00 \\
(0.95,1.06)\end{array}$ & $\begin{array}{c}1.09 \\
(0.96,1.23) \\
0.95 \\
(0.85,1.06)\end{array}$ \\
\hline $0-64$ years & $\begin{array}{c}1.05 \\
(0.80,1.38)\end{array}$ & $\begin{array}{c}0.88 \\
(0.66,1.17)\end{array}$ & $\begin{array}{c}0.74 \\
(0.59,0.92)\end{array}$ & $\begin{array}{c}0.90 \\
(0.64,1.27)\end{array}$ & $\begin{array}{c}1.17 \\
(0.93,1.47)\end{array}$ & $\begin{array}{c}0.91 \\
(0.68,1.21)\end{array}$ & $\begin{array}{c}0.85 \\
(0.71,1.02)\end{array}$ & $\begin{array}{c}0.93 \\
(0.67,1.31)\end{array}$ & $\begin{array}{c}1.22 \\
(1.001 \\
1.49)\end{array}$ & $\begin{array}{c}1.32 \\
(0.92,1.89)\end{array}$ & $\begin{array}{c}1.03 \\
(0.87,1.23)\end{array}$ & $\begin{array}{c}0.97 \\
(0.66,1.43)\end{array}$ \\
\hline $\begin{array}{l}65 \text { years and over } \\
\text { Cardiac Arrest }\end{array}$ & $\begin{array}{c}0.92 \\
(0.83,1.03)\end{array}$ & $\begin{array}{c}1.03 \\
(0.92,1.15)\end{array}$ & $\begin{array}{c}0.84 \\
(0.77,0.92)\end{array}$ & $\begin{array}{c}0.96 \\
(0.83,1.08)\end{array}$ & $\begin{array}{c}0.96 \\
(0.87,1.06)\end{array}$ & $\begin{array}{c}0.98 \\
(0.88,1.10)\end{array}$ & $\begin{array}{c}0.89 \\
(0.83,0.97)\end{array}$ & $\begin{array}{c}0.94 \\
(0.82,1.08)\end{array}$ & $\begin{array}{c}0.97 \\
(0.90,1.06)\end{array}$ & $\begin{array}{c}0.97 \\
(0.83,1.13)\end{array}$ & $\begin{array}{c}0.93 \\
(0.87,1.01)\end{array}$ & $\begin{array}{c}0.92 \\
(0.78,1.07)\end{array}$ \\
\hline $0-64$ years & $\begin{array}{c}0.90 \\
(0.52,1.58)\end{array}$ & $\begin{array}{c}0.83 \\
(0.47,1.45)\end{array}$ & $\begin{array}{c}1.31 \\
(0.84,2.04)\end{array}$ & $\begin{array}{c}0.78 \\
(0.40,1.52)\end{array}$ & $\begin{array}{c}0.99 \\
(0.60,1.62)\end{array}$ & $\begin{array}{c}1.14 \\
(0.67,1.97)\end{array}$ & $\begin{array}{c}1.30 \\
(0.89,1.89)\end{array}$ & $\begin{array}{c}1.08 \\
(0.56,2.08)\end{array}$ & $\begin{array}{c}0.96 \\
(0.62,1.49)\end{array}$ & $\begin{array}{c}1.35 \\
(0.68,2.67)\end{array}$ & $\begin{array}{c}1.25 \\
(0.88,1.77)\end{array}$ & $\begin{array}{c}1.35 \\
(0.63,2.90)\end{array}$ \\
\hline 65 years and over & $\begin{array}{c}1.19 \\
(0.71,2.01)\end{array}$ & $\begin{array}{c}1.11 \\
(0.70,1.78)\end{array}$ & $\begin{array}{c}0.91 \\
(0.62,1.34)\end{array}$ & $\begin{array}{c}1.85^{*} \\
(1.08,3.16)\end{array}$ & $\begin{array}{c}0.78 \\
(0.48,1.25)\end{array}$ & $\begin{array}{c}1.28 \\
(0.80,2.05)\end{array}$ & $\begin{array}{c}0.93 \\
(0.65,1.32)\end{array}$ & $\begin{array}{c}1.65 * \\
(0.96,2.84)\end{array}$ & $\begin{array}{c}0.90 \\
(0.58,1.38)\end{array}$ & $\begin{array}{c}0.96 \\
(0.48,1.89)\end{array}$ & $\begin{array}{c}1.18 \\
(0.84,1.67)\end{array}$ & $\begin{array}{c}1.16 \\
(0.59,2.29)\end{array}$ \\
\hline $0-64$ years & $\begin{array}{c}0.91 \\
(0.78,1.06)\end{array}$ & $\begin{array}{c}0.99 \\
(0.86,1.14)\end{array}$ & $\begin{array}{c}1.13 \\
(1.01,1.27)\end{array}$ & $\begin{array}{c}0.91 * * \\
(0.77,1.09)\end{array}$ & $\begin{array}{c}0.96 \\
(0.85,1.05)\end{array}$ & $\begin{array}{c}1.07 \\
(0.93,1.24)\end{array}$ & $\begin{array}{c}1.07 \\
(0.97,1.18)\end{array}$ & $\begin{array}{c}0.91 \\
(0.76,1.08)\end{array}$ & $\begin{array}{c}0.96 \\
(0.86,1.06)\end{array}$ & $\begin{array}{c}0.95 \\
(0.79,1.15)\end{array}$ & $\begin{array}{c}1.03 \\
(0.94,1.13)\end{array}$ & $\begin{array}{c}0.84 \\
(0.69,1.03)\end{array}$ \\
\hline 65 years and over & $\begin{array}{c}0.97 \\
(0.86,1.09)\end{array}$ & $\begin{array}{c}1.02 \\
(0.91,1.15)\end{array}$ & $\begin{array}{c}0.91 \\
(0.83,0.998)\end{array}$ & $\begin{array}{c}0.95 \\
(0.83,1.09)\end{array}$ & $\begin{array}{c}0.99 \\
(0.90,1.09)\end{array}$ & $\begin{array}{c}0.99 \\
(0.88,1.11)\end{array}$ & $\begin{array}{c}0.94 \\
(0.87,1.01)\end{array}$ & $\begin{array}{c}1.01 \\
(0.89,1.16)\end{array}$ & $\begin{array}{c}1.03 \\
(0.95,1.12)\end{array}$ & $\begin{array}{c}0.97 \\
(0.83,1.13)\end{array}$ & $\begin{array}{c}1.07 \\
(1.00,1.15)\end{array}$ & $\begin{array}{c}1.05 \\
(0.90,1.23)\end{array}$ \\
\hline Conduction Disorc & & & & & & & & & & & & \\
\hline $0-64$ years & $\begin{array}{c}0.80 \\
(0.41,1.54)\end{array}$ & $\begin{array}{c}1.03 \\
(0.52,2.02)\end{array}$ & $\begin{array}{c}1.26 \\
(0.77,2.06)\end{array}$ & $\begin{array}{c}1.80 \\
(0.83,4.01)\end{array}$ & $\begin{array}{c}0.91 \\
(0.54,1.53)\end{array}$ & $\begin{array}{c}1.21 \\
(0.63,2.35)\end{array}$ & $\begin{array}{c}1.24 \\
(0.81,1.91)\end{array}$ & $\begin{array}{c}1.36 \\
(0.61,3.04)\end{array}$ & $\begin{array}{c}1.07 \\
(0.67,1.71)\end{array}$ & $\begin{array}{c}1.22 \\
(0.53,2.78)\end{array}$ & $\begin{array}{c}1.06 \\
(0.72,1.57)\end{array}$ & $\begin{array}{c}1.20 \\
(0.52,2.80)\end{array}$ \\
\hline 65 years and over & $\begin{array}{c}0.84 \\
(0.61,1.16)\end{array}$ & $\begin{array}{c}1.04 \\
(0.74,1.47)\end{array}$ & $\begin{array}{c}0.83 \\
(0.65,1.07)\end{array}$ & $\begin{array}{c}0.77 \\
(0.51,1.16)\end{array}$ & $\begin{array}{c}0.89 \\
(0.68,1.16)\end{array}$ & $\begin{array}{c}0.89 \\
(0.63,1.27)\end{array}$ & $\begin{array}{c}0.87 \\
(0.70,1.08)\end{array}$ & $\begin{array}{c}0.76 \\
(0.50,1.16)\end{array}$ & $\begin{array}{c}1.12 \\
(0.89,1.41)\end{array}$ & $\begin{array}{c}0.81 \\
(0.52,1.26)\end{array}$ & $\begin{array}{c}0.84 \\
(0.68,1.04)\end{array}$ & $\begin{array}{c}0.77 \\
(0.48,1.24)\end{array}$ \\
\hline Hypertensive Dise & & & & & & & & & & & & \\
\hline $0-64$ years & $\begin{array}{c}0.89 \\
(0.58,1.36)\end{array}$ & $\begin{array}{c}0.96 \\
(0.62,1.48)\end{array}$ & $\begin{array}{c}0.89 \\
(0.65,1.24)\end{array}$ & $\begin{array}{c}0.93 \\
(0.55,1.56)\end{array}$ & $\begin{array}{c}0.91 \\
(0.63,1.31)\end{array}$ & $\begin{array}{c}1.16 \\
(0.75,1.78)\end{array}$ & $\begin{array}{c}1.06 \\
(0.80,1.39)\end{array}$ & $\begin{array}{c}1.22 \\
(0.73,2.03)\end{array}$ & $\begin{array}{c}1.11 \\
(0.81,1.52)\end{array}$ & $\begin{array}{c}1.88 \\
(1.07,3.28)\end{array}$ & $\begin{array}{c}1.08 \\
(0.83,1.40)\end{array}$ & $\begin{array}{c}1.82 \\
(1.03,3.20)\end{array}$ \\
\hline 65 years and over & $\begin{array}{c}0.84 \\
(0.59,1.21)\end{array}$ & $\begin{array}{c}0.82 \\
(0.56,1.19)\end{array}$ & $\begin{array}{c}1.11 \\
(0.86,1.44)\end{array}$ & $\begin{array}{c}0.92 \\
(0.58,1.44)\end{array}$ & $\begin{array}{c}0.87 \\
(0.65,1.18)\end{array}$ & $\begin{array}{c}0.62 \\
(0.42,0.93)\end{array}$ & $\begin{array}{c}0.82 \\
(0.65,1.05)\end{array}$ & $\begin{array}{c}0.74 \\
(0.46,1.18)\end{array}$ & $\begin{array}{c}0.79 \\
(0.61,1.02)\end{array}$ & $\begin{array}{c}0.74 \\
(0.43,1.25)\end{array}$ & $\begin{array}{c}0.74 \\
(0.59,0.92)\end{array}$ & $\begin{array}{c}1.02 \\
(0.62,1.68)\end{array}$ \\
\hline
\end{tabular}

${ }^{*}$ Denotes a statistically significant positive interaction term $p$-value $(<0.05) .{ }^{* *}$ Denotes a statistically significant negative interaction term $p$-value $(<0.05)$. 


\section{Discussion}

This study examined whether $\mathrm{PM}_{10}$ modifies the association between heat waves and EHAs for six cardiovascular diseases in Greater Sydney, Australia. We estimated and compared the effect of heat waves on high- and low-level $\mathrm{PM}_{10}$ days at $\operatorname{lag}_{0}-\operatorname{lag}_{2}$ for three age groups: all ages combined, 0-64 years, and 65 years and above, and tested the sensitivity of three heat wave definitions. We found some evidence that $\mathrm{PM}_{10}$ modifies the association between heat waves and EHAs for certain cardiovascular diseases. Stronger heat wave effects were observed on high- compared to low-level $\mathrm{PM}_{10}$ days for EHAs for cardiac arrest for all three age groups; conduction disorders for 0-64 years; and hypertensive diseases for all ages combined and 0-64 years. These findings, however, were generally not consistent across all heat wave definitions and lags. Positive, statistically significant interactions were found only for EHAs for hypertensive diseases (all ages combined) and cardiac arrest (65 years and above).

It is difficult to directly compare our findings to previous studies, as most of the work to date examining the potential interactive effects of temperature or heat waves and $\mathrm{PM}_{10}$ on cardiovascular health outcomes has considered cardiovascular mortality (e.g., [26,27,30,32-34]). Few studies have considered cardiovascular morbidity as the health outcome, particularly cause-specific cardiovascular morbidity $[36,54,55]$. Much like our findings, the results of the studies considering cardiovascular morbidity have been broadly inconsistent, although different exposure variables have been considered (i.e., temperature, season, and relative humidity). For example, Ren et al. [36] found evidence of a statistical interaction between temperature and total cardiovascular hospital admissions at different lags in Brisbane, Australia, but found no such evidence for total cardiovascular emergency presentations. Qiu et al. [55] reported that the association between $\mathrm{PM}_{10}$ and emergency hospital admissions for ischemic heart disease was strongest in the cool season and at lower levels of relative humidity in Hong Kong, China. Further, Kang et al. [7] found no evidence of a significant interactive effect between heat waves and $\mathrm{PM}_{10}$ on out-of-hospital cardiac arrest in Korea, which is in general disagreement with our findings regarding EHAs for cardiac arrest. The level and source composition of $\mathrm{PM}_{10}$ differs across regions and cities [56-58], as does population acclimatisation to temperature changes and heat waves $[1,59]$. It is therefore important to conduct further localised studies to account for these differences and clarify our understanding of any potential interactive effects of these environmental exposures on cardiovascular morbidity.

It is plausible that air pollution and heat exposure may interact on a biological level, although the exact causal pathways and mechanisms involved are not known. The activation of the body's thermoregulatory system and mechanisms during heat stress can facilitate and increase the absorption and entry of toxins and air pollutants into the body, as well as alter the body's response to such substances [24]. The strength of the toxicity of a chemical or toxin on a biological system can be exacerbated by increased body temperature [24,60]. Passive heat exposure can stress the cardiovascular system, where increased skin blood flow during thermoregulation results in increased cardiac output, which in turn is mediated by increases in heart rate [61]. Madaniyazi et al. [62] observed a "V" shaped relationship between mean temperature and heart rate and blood pressure (systolic and diastolic) in Chinese adults, finding heat effects above certain thresholds. Others have, however, observed a decrease in systolic blood pressure with an increase in ambient temperature [63]. Ren et al. [64] found that increased ambient temperature is associated with decreased heart rate variability (HRV) during the warm season, but found no evidence of an interactive effect between ambient temperature and $\mathrm{PM}_{2.5}$ on HRV. Particulate matter may also adversely affect the cardiovascular system by directly entering into the systemic circulation (smaller particles: $\mathrm{PM}_{2.5}$ or $\mathrm{PM}_{1.0}$ ), or indirectly by affecting the autonomic nervous system or inducing an inflammatory response [65]. Stafoggia et al. [26] noted that their findings of stronger $\mathrm{PM}_{10}$ effects on mortality during the warm season might be a result of increased exposure to this pollutant, with individuals more likely to open their windows and spend time outdoors during the summer months.

We observed positive, statistically significant interactions between heat wave and high-level $\mathrm{PM}_{10}$ days on EHAs for cardiac arrest among the elderly. Previous studies examining the susceptibility of 
specific age groups to the potential interactive effects of high temperatures or heat waves and $\mathrm{PM}_{10}$ on cardiovascular mortality have generally found effect modification to be more pronounced among the elderly $[27,31,33]$. The elderly are particularly susceptible to extreme heat exposure due to their decreased capacity to effectively thermoregulate, with sweat gland output, blood flow to the skin, and cardiac output reduced [66]. Given the general decline of the body's physiological processes with age and the higher prevalence of cardiovascular diseases among older age groups, the elderly are also susceptible to the adverse effects of particulate matter [67]. We also found some evidence of effect modification in the younger age group for certain diseases. The reasons for this are unclear, although it may be because younger populations are generally more physically active than older populations [68], which may result in more time spent outdoors, subsequently increasing their exposure levels.

We found positive, statistically significant interactions at $\operatorname{lag}_{1}$ and $\operatorname{lag}_{2}$ for certain cardiovascular diseases, but not at $\operatorname{lag}_{0}$. Evidence of an interactive effect between high temperature and high-levels of $\mathrm{PM}_{10}$ on cardiovascular health outcomes has also been found at certain lags [25,36]. For example, Qian et al. [25] observed stronger $\mathrm{PM}_{10}$ effects on cardiovascular mortality at high compared to normal level temperatures at $\operatorname{lag}_{0-1}$ in Wuhan, China. Short lag effects have also been observed when examining the independent effects of high temperatures and $\mathrm{PM}_{10}$ on cardiovascular morbidity $[3,15]$. Positive, statistically significant interactions were also found for some heat wave definitions only. The choice of heat wave definition has been shown to affect both the magnitude and statistical significance of the association between heat waves and health outcomes [69]. Each of the three heat wave definitions used in this study identified different days as "exposure" days, and the total number of exposure days varied between our definitions (See Table 3). It is likely that this affected our models, as well as the calculation of the interaction term between heat wave and high-level $\mathrm{PM}_{10}$ days. It is also possible that different temperature metrics (maximum, mean, minimum) may have different impacts on cardiovascular health outcomes, although differences in their interaction with $\mathrm{PM}_{10}$ is unclear. For example, Kang et al. [7] found that the risk of out-of-hospital cardiac arrest during heat waves was highest in the afternoon ( 3 p.m. to 5 p.m.), which coincided with the peak of daily outdoor temperature.

A few negative, statistically significant interactions were found, and negative associations were observed across both high- and low-level $\mathrm{PM}_{10}$ days and in Figure 1 for certain cardiovascular diseases. Several previous studies have also found null or negative associations between increased temperature or extreme heat and hospital admissions for cardiovascular diseases $[8,9,11]$. Such findings are in contrast to the positive associations often observed between high temperature or heat waves and cardiovascular mortality across several regions, particularly among the elderly [70,71]. The exact reasons for the differences found between these cardiovascular health outcomes are not known. One possible explanation is that individuals may die quickly from cardiovascular disease during high temperatures before they are able to seek medical attention or be admitted to hospital [72].

This study has some potential strengths. To the best of our knowledge, this is the first study to examine the potential interactive effects of heat waves and $\mathrm{PM}_{10}$ on cause-specific cardiovascular hospital admissions in an Australian city. By examining and comparing six specific cardiovascular diseases, we have shown that some conditions may be more susceptible to the potential interactive effects of heat waves and $\mathrm{PM}_{10}$ than others (e.g., cardiac arrest). We also analysed a relatively long period of time series data (12 years) and controlled for other ambient air pollutants including ozone and nitrogen dioxide.

This study has some potential limitations. The analysis was performed for a single city and, therefore, our results may not be generalisable given that $\mathrm{PM}_{10}$ levels and mixtures can vary geographically, as well as population acclimatisation to heat waves. The samples sizes for some of the cardiovascular diseases were relatively small when stratified by age group (e.g., cardiac arrest, conductions disorders), and we had limited power to detect interaction effects because of the small number of days that were classified as being heatwaves and having high $\mathrm{PM}_{10}$ levels. Therefore, caution is warranted when interpreting the significance of these results. We estimated exposure to heat waves and $\mathrm{PM}_{10}$ by calculating the daily city-wide average using monitoring stations, and not by measuring 
an individual's personal exposure level, which may have resulted in some exposure misclassification. Our analysis did not account for transfers between episodes of care in the hospital admissions data, and thus it is possible that admissions relating to the same cardiac event for an individual were counted as different events. Further, heat wave forecasts or government-issued heat wave warnings may result in individuals exhibiting avoidance behaviours, especially for people with existing health conditions. This individual level response is beyond the scope of this research.

\section{Conclusions}

This study found some evidence that $\mathrm{PM}_{10}$ modifies the association between heat waves and hospital admissions for certain cardiovascular diseases. Our findings, however, showed inconsistencies and largely differed across age group, disease, lag, and heat wave definition. Given the differences found across diseases, our study highlights the need for future studies to consider, where possible, cause-specific outcomes when examining the potential interactive effects of heat waves and ambient air pollution. With both heat waves and levels of ambient particulate matter expected to increase under climate change, it is important to consider potential effect modification by air pollution when examining the impacts of heat waves on cardiovascular morbidity. As our study has shown, this is true even for locations with comparatively low levels of particulate matter, such as Australia.

Author Contributions: Data curation M.P. and Y.Z.; formal analysis M.P. and A.H.; methodology, M.P. and A.H.; writing-original draft preparation, M.P.; writing-review and editing, D.G. and A.H.; supervision, D.G. and A.H.; project administration, D.G.; investigation, A.H.; resources, D.G.

Funding: This research received no external funding.

Acknowledgments: The authors would like to thank and acknowledge the Australian Bureau of Meteorology for providing the meteorological data; the NSW Office of Environment and Heritage for providing the air population data and the Centre for Epidemiology and Evidence, NSW Ministry of Health for providing the hospital admissions data from the Admitted Patient Data Collection (SAPHaRI). Further, the authors would like to thank Associate Professor Lisa Alexander for her advice and insights regarding weather station data and temperature extremes; Professor Adrian Barnett for his assistance with the application of the "season" package in the R Statistical Computing Environment and James Goldie for his assistance and insights regarding public holiday data.

Conflicts of Interest: The authors declare no conflict of interest.

\section{Appendix A}

Table A1. The effect of heat wave days on "emergency" hospital admissions (EHAs) for six cardiovascular diseases on days with high levels of particulate matter $\left(\mathrm{PM}_{10}\right)$ compared to days with low levels of $\mathrm{PM}_{10}$ in the Sydney Statistical Division (SSD) during the warm season, 2001 to 2013, for all ages at $\operatorname{lag}_{2}$.

\begin{tabular}{|c|c|c|c|c|c|c|}
\hline \multirow[b]{2}{*}{$\begin{array}{l}\text { Cardiovascular } \\
\text { Disease }\end{array}$} & \multicolumn{2}{|c|}{ HWD01 } & \multicolumn{2}{|c|}{ HWD02 } & \multicolumn{2}{|c|}{ HWD03 } \\
\hline & $\begin{array}{l}\text { Heat Effect } \\
\text { Low } \text { PM }_{10}\end{array}$ & $\begin{array}{l}\text { Heat Effect } \\
\text { High } \text { PM }_{10}\end{array}$ & $\begin{array}{c}\text { Heat Effect } \\
\text { Low } \text { PM }_{10}\end{array}$ & $\begin{array}{l}\text { Heat Effect } \\
\text { High } \mathbf{P M}_{10}\end{array}$ & $\begin{array}{l}\text { Heat Effect } \\
\text { Low } \text { PM }_{10}\end{array}$ & $\begin{array}{l}\text { Heat Effect } \\
\text { High } \mathbf{P M}_{10}\end{array}$ \\
\hline $\begin{array}{l}\text { Ischemic Heart } \\
\text { Disease }\end{array}$ & $\begin{array}{c}1.02 \\
(0.98,1.07)\end{array}$ & $\begin{array}{c}0.93^{* *} \\
(0.85,1.02)\end{array}$ & $\begin{array}{c}1.00 \\
(0.96,1.04)\end{array}$ & $\begin{array}{c}0.95 \\
(0.87,1.03)\end{array}$ & $\begin{array}{c}0.99 \\
(0.96,1.03)\end{array}$ & $\begin{array}{c}0.98 \\
(0.89,1.07)\end{array}$ \\
\hline Heart Failure & $\begin{array}{c}0.87 \\
(0.81,0.94)\end{array}$ & $\begin{array}{c}0.89 \\
(0.76,1.03)\end{array}$ & $\begin{array}{c}0.92 \\
(0.86,0.99)\end{array}$ & $\begin{array}{c}0.89 \\
(0.76,1.03)\end{array}$ & $\begin{array}{c}0.94 \\
(0.88,1.002)\end{array}$ & $\begin{array}{c}0.91 \\
(0.77,1.07)\end{array}$ \\
\hline Cardiac Arrest & $\begin{array}{c}1.13 \\
(0.87,1.47)\end{array}$ & $\begin{array}{c}1.26 \\
(0.75,2.12)\end{array}$ & $\begin{array}{c}1.13 \\
(0.89,1.45)\end{array}$ & $\begin{array}{c}1.37 \\
(0.89,2.09)\end{array}$ & $\begin{array}{c}1.13 \\
(0.89,1.43)\end{array}$ & $\begin{array}{c}0.73 \\
(0.40,1.35)\end{array}$ \\
\hline Heart Arrhythmia & $\begin{array}{c}0.98 \\
(0.92,1.05)\end{array}$ & $\begin{array}{c}1.00 \\
(0.87,1.14)\end{array}$ & $\begin{array}{c}1.00 \\
(0.95,1.06)\end{array}$ & $\begin{array}{c}1.04 \\
(0.92,1.19)\end{array}$ & $\begin{array}{c}1.02 \\
(0.97,1.08)\end{array}$ & $\begin{array}{c}1.08 \\
(0.94,1.24)\end{array}$ \\
\hline Conduction Disorders & $\begin{array}{c}1.01 \\
(0.82,1.23)\end{array}$ & $\begin{array}{c}0.81 \\
(0.51,1.28)\end{array}$ & $\begin{array}{c}0.92 \\
(0.76,1.11)\end{array}$ & $\begin{array}{c}0.88 \\
(0.57,1.35)\end{array}$ & $\begin{array}{c}0.85 \\
(0.71,1.01)\end{array}$ & $\begin{array}{c}0.90 \\
(0.58,1.39)\end{array}$ \\
\hline Hypertensive Disease & $\begin{array}{c}1.02 \\
(0.85,1.23)\end{array}$ & $\begin{array}{c}0.94 \\
(0.62,1.41)\end{array}$ & $\begin{array}{c}1.02 \\
(0.86,1.20)\end{array}$ & $\begin{array}{c}0.91 \\
(0.61,1.35)\end{array}$ & $\begin{array}{c}1.00 \\
(0.85,1.17)\end{array}$ & $\begin{array}{c}1.11 \\
(0.73,1.68)\end{array}$ \\
\hline
\end{tabular}

** Denotes a statistically significant negative interaction term $p$-value $(<0.05)$. 
Table A2. The effect of heat wave days on EHAs for cardiovascular diseases on days with high levels of $\mathrm{PM}_{10}$ compared to days with low levels of $\mathrm{PM}_{10}$ in the SSD during the warm season, 2001 to 2013, for those aged 0-64 years and 65 years and over at $\operatorname{lag}_{2}$.

\begin{tabular}{|c|c|c|c|c|c|c|}
\hline \multirow[b]{2}{*}{$\begin{array}{l}\text { Cardiovascular } \\
\text { Disease }\end{array}$} & \multicolumn{2}{|c|}{ HWD01 } & \multicolumn{2}{|c|}{ HWD02 } & \multicolumn{2}{|c|}{ HWD03 } \\
\hline & $\begin{array}{c}\text { Heat Effect } \\
\text { Low } \mathrm{PM}_{10}\end{array}$ & $\begin{array}{l}\text { Heat Effect } \\
\text { High } \mathbf{P M}_{10}\end{array}$ & $\begin{array}{c}\text { Heat Effect } \\
\text { Low } \text { PM }_{10}\end{array}$ & $\begin{array}{l}\text { Heat Effect } \\
\text { High } \mathbf{P M}_{10}\end{array}$ & $\begin{array}{l}\text { Heat Effect } \\
\text { Low } \mathbf{P M}_{10}\end{array}$ & $\begin{array}{l}\text { Heat Effect } \\
\text { High } \text { PM }_{10}\end{array}$ \\
\hline \multicolumn{7}{|l|}{ Ischemic Heart Disease } \\
\hline $0-64$ years & $\begin{array}{c}1.07 \\
(1.00,1.14)\end{array}$ & $\begin{array}{c}0.96 \\
(0.84,1.11)\end{array}$ & $\begin{array}{c}1.01 \\
(0.95,1.07)\end{array}$ & $\begin{array}{c}0.95 \\
(0.84,1.09)\end{array}$ & $\begin{array}{c}1.00 \\
(0.94,1.06)\end{array}$ & $\begin{array}{c}1.04 \\
(0.86,1.14)\end{array}$ \\
\hline 65 years and over & $\begin{array}{c}0.99 \\
(0.94,1.05)\end{array}$ & $\begin{array}{c}0.91 \\
(0.80,1.02)\end{array}$ & $\begin{array}{c}0.99 \\
(0.94,1.05)\end{array}$ & $\begin{array}{c}0.94 \\
(0.84,1.06)\end{array}$ & $\begin{array}{c}0.99 \\
(0.94,1.04)\end{array}$ & $\begin{array}{c}0.93 \\
(0.82,1.06)\end{array}$ \\
\hline \multicolumn{7}{|l|}{ Heart Failure } \\
\hline $0-64$ years & $\begin{array}{c}0.84 \\
(0.69,1.02)\end{array}$ & $\begin{array}{c}0.78 \\
(0.51,1.19)\end{array}$ & $\begin{array}{c}0.90 \\
(0.75,1.07)\end{array}$ & $\begin{array}{c}1.02 \\
(0.69,1.49)\end{array}$ & $\begin{array}{c}0.99 \\
(0.84,1.18)\end{array}$ & $\begin{array}{c}0.92 \\
(0.60,1.40)\end{array}$ \\
\hline 65 years and over & $\begin{array}{c}0.90 \\
(0.83,0.98)\end{array}$ & $\begin{array}{c}0.90 \\
(0.75,1.05)\end{array}$ & $\begin{array}{c}0.92 \\
(0.86,0.99)\end{array}$ & $\begin{array}{c}0.86 \\
(0.73,1.02)\end{array}$ & $\begin{array}{c}0.93 \\
(0.87,0.997)\end{array}$ & $\begin{array}{c}0.91 \\
(0.76,1.08)\end{array}$ \\
\hline \multicolumn{7}{|l|}{ Cardiac Arrest } \\
\hline $0-64$ years & $\begin{array}{c}1.51 \\
(1.01,2.26)\end{array}$ & $\begin{array}{c}0.76 \\
(0.31,1.85)\end{array}$ & $\begin{array}{c}1.33 \\
(0.93,1.91)\end{array}$ & $\begin{array}{c}0.77 \\
(0.34,1.74)\end{array}$ & $\begin{array}{c}1.17 \\
(0.83,1.65)\end{array}$ & $\begin{array}{c}0.61 \\
(0.24,1.57)\end{array}$ \\
\hline 65 years and over & $\begin{array}{c}0.92 \\
(0.65,1.31)\end{array}$ & $\begin{array}{c}1.71^{*} \\
(0.89,3.28)\end{array}$ & $\begin{array}{c}0.98 \\
(0.70,1.38)\end{array}$ & $\begin{array}{c}1.68 \\
(0.89,3.18)\end{array}$ & $\begin{array}{c}1.10 \\
(0.80,1.51)\end{array}$ & $\begin{array}{c}0.83 \\
(0.36,1.90)\end{array}$ \\
\hline Heart Arrhythmia & & & & & & \\
\hline $0-64$ years & $\begin{array}{c}1.05 \\
(0.95,1.16)\end{array}$ & $\begin{array}{c}1.04 \\
(0.84,1.29)\end{array}$ & $\begin{array}{c}1.03 \\
(0.94,1.13)\end{array}$ & $\begin{array}{c}1.00 \\
(0.81,1.22)\end{array}$ & $\begin{array}{c}0.98 \\
(0.89,1.07)\end{array}$ & $\begin{array}{c}0.96 \\
(0.76,1.20)\end{array}$ \\
\hline 65 years and over & $\begin{array}{c}0.95 \\
(0.87,1.03)\end{array}$ & $\begin{array}{c}0.97 \\
(0.82,1.15)\end{array}$ & $\begin{array}{c}0.99 \\
(0.92,1.07)\end{array}$ & $\begin{array}{c}1.08 \\
(0.91,1.27)\end{array}$ & $\begin{array}{c}1.05 \\
(0.98,1.12)\end{array}$ & $\begin{array}{c}1.16 \\
(0.97,1.39)\end{array}$ \\
\hline Conduction Disorders & & & & & & \\
\hline $0-64$ years & $\begin{array}{c}1.09 \\
(0.70,1.72)\end{array}$ & $\begin{array}{c}2.07 \\
(0.79,5.42)\end{array}$ & $\begin{array}{c}1.08 \\
(0.72,1.62)\end{array}$ & $\begin{array}{c}1.80 \\
(0.70,4.66)\end{array}$ & $\begin{array}{c}0.81 \\
(0.55,1.18)\end{array}$ & $\begin{array}{c}1.58 \\
(0.59,4.22)\end{array}$ \\
\hline 65 years and over & $\begin{array}{c}0.99 \\
(0.79,1.24)\end{array}$ & $\begin{array}{c}0.62 \\
(0.36,1.07)\end{array}$ & $\begin{array}{c}0.88 \\
(0.72,1.09)\end{array}$ & $\begin{array}{c}0.73 \\
(0.45,1.19)\end{array}$ & $\begin{array}{c}0.86 \\
(0.71,1.06)\end{array}$ & $\begin{array}{c}0.78 \\
(0.47,1.27)\end{array}$ \\
\hline Hypertensive Disease & & & & & & \\
\hline $0-64$ years & $\begin{array}{c}0.83 \\
(0.62,1.11)\end{array}$ & $\begin{array}{c}1.08 \\
(0.60,1.94)\end{array}$ & $\begin{array}{c}1.06 \\
(0.82,1.38)\end{array}$ & $\begin{array}{c}1.21 \\
(0.69,2.14)\end{array}$ & $\begin{array}{c}1.00 \\
(0.78,1.28)\end{array}$ & $\begin{array}{c}1.64 \\
(0.91,2.98)\end{array}$ \\
\hline 65 years and over & $\begin{array}{c}1.18 \\
(0.93,1.50)\end{array}$ & $\begin{array}{c}0.77 \\
(0.43,1.40)\end{array}$ & $\begin{array}{c}0.98 \\
(0.79,1.22)\end{array}$ & $\begin{array}{c}0.69 \\
(0.39,1.21)\end{array}$ & $\begin{array}{c}0.99 \\
(0.81,1.22)\end{array}$ & $\begin{array}{c}0.78 \\
(0.43,1.42)\end{array}$ \\
\hline
\end{tabular}

* Denotes a statistically significant positive interaction term $p$-value $(<0.05)$.

\section{References}

1. Nichols, M.; Peterson, K.; Alston, L.; Allender, S. Australian Heart Disease Statistics 2014; National Heart Foundation of Australia: Melbourne, Australia, 2014.

2. World Health Organization. Cardiovascular diseases (CVDs). 2017. Available online: http://www.who.int/ mediacentre/factsheets/fs317/en/ (accessed on 2 February 2018).

3. Lin, S.; Luo, M.; Walker, R.J.; Liu, X.; Hwang, S.; Chinery, R. Extreme high temperatures and hospital admissions for respiratory and cardiovascular diseases. Epidemiology 2009, 20, 738-746. [CrossRef]

4. Ostro, B.; Rauch, S.; Green, R.; Malig, B.; Basu, R. The effects of temperature and use of air conditioning on hospitalizations. Am. J. Epidemiol. 2010, 172, 1053-1061. [CrossRef]

5. Ma, W.; Xu, X.; Peng, L.; Kan, H. Impact of extreme temperature on hospital admission in Shanghai, China. Sci. Total Environ. 2011, 409, 3634-3637. [CrossRef]

6. Nitschke, M.; Tucker, G.R.; Bi, P. Morbidity and mortality during heatwaves in metropolitan Adelaide. Med. J. Aust. 2007, 187, 662-665.

7. Kang, S.H.; Oh, I.Y.; Heo, J.; Lee, H.; Kim, J.; Lim, W.H.; Cho, Y.; Choi, E.K.; Yi, S.M.; Shin, S.D.; et al. Heat, heat waves, and out-of-hospital cardiac arrest. Int. J. Cardiol. 2016, 221, 232-237. [CrossRef]

8. Michelozzi, P.; Accetta, G.; De Sario, M.; D’Ippoliti, D.; Marino, C.; Baccini, M.; Biggeri, A.; Anderson, H.R.; Katsouyanni, K.; Ballester, F.; et al. High temperature and hospitalizations for cardiovascular and respiratory causes in 12 European cites. Am. J. Respir. Crit. Care Med. 2009, 179, 383-389. [CrossRef]

9. Turner, L.R.; Barnett, A.G.; Connell, D.; Tong, S. Ambient temperature and cardiorespiratory morbidity: A systematic review and meta-analysis. Epidemiology 2012, 23, 594-606. [CrossRef] 
10. Phung, D.; Thai, P.K.; Guo, Y.; Morawska, L.; Rutherford, S.; Chu, C. Ambient temperature and risk of cardiovascular hospitalization: An updated systematic review and meta-analysis. Sci. Total Environ. 2016, 550, 1084-1102. [CrossRef]

11. Ogbomo, A.S.; Gronlund, C.J.; O’Neill, M.S.; Konen, T.; Cameron, L.; Wahl, R. Vulnerability to extreme-heat-associated hospitalization in three counties in Michigan, USA, 2000-2009. Int. J. Biometeorol. 2017, 61, 833-843. [CrossRef]

12. US EPA (United States Environment Protection Authority). Particulate Matter (PM) Pollution. 2016. Available online: https://www.epa.gov/pm-pollution/particulate-matter-pm-basics\#PM (accessed on 6 September 2017).

13. Keywood, M.D.; Emmerson, K.M.; Hibberd, M.F. Ambient Air Quality: Coarse Particulate Matter (PM10). In Australia State of the Environment 2016; 2016. Available online: https://soe.environment.gov.au/theme/ ambient-air-quality/topic/2016/coarse-particulate-matter-pm10 (accessed on 17 April 2018).

14. Barnett, A.G.; Williams, G.M.; Schwartz, J.; Best, T.L.; Neller, A.H.; Petroeschevsky, A.L.; Simpson, R.W. The effects of air pollution on hospitalizations for cardiovascular disease in elderly people in Australian and New Zealand cities. Environ. Health Perspect. 2006, 114, 1018-1023. [CrossRef]

15. Colais, P.; Faustini, A.; Stafoggia, M.; Berti, G.; Bisanti, L.; Cadum, E.; Cernigliaro, A.; Mallone, S.; Pacelli, B.; Serinelli, M.; et al. Particulate air pollution and hospital admissions for cardiac diseases in potentially sensitive subgroups. Epidemiology 2012, 23, 473-481. [CrossRef]

16. Stafoggia, M.; Samoli, E.; Alessandrini, E.; Cadum, E.; Ostro, B.; Berti, G.; Faustini, A.; Jacquemin, B.; Linares, C.; Pascal, M.; et al. Short-term associations between fine and coarse particulate matter and hospitalizations in Southern Europe: Results from the MED-PARTICLES Project. Environ. Health Perspect. 2013, 121, 1026-1033. [CrossRef]

17. Schwartz, J.; Morris, R. Air pollution and hospital admissions for cardiovascular disease in Detroit, Michigan. Am. J. Epidemiol. 1995, 142, 23-35. [CrossRef]

18. Xu, A.; Mu, Z.; Jiang, B.; Wang, W.; Yu, H.; Zhang, L.; Li, J. Acute effects of particulate air pollution in ischemic heart disease hospitalizations in Shanghai, China. Int. J. Environ. Res. Public Health 2017, 14, 168. [CrossRef]

19. Shah, A.S.V.; Langrish, J.P.; Nair, H.; McAllister, D.A.; Hunter, A.L.; Donaldson, K.; Newby, D.E.; Mills, N.L. Global association of air pollution and heart failure: A systematic review and meta-analysis. Lancet 2013, 382, 1039-1048. [CrossRef]

20. Zhao, R.; Chen, S.; Wang, W.; Huang, J.; Wang, K.; Liu, L.; Wei, S. The impact of short-term exposure to air pollutants on the onset of out-of-hospital cardiac arrest: A systematic review and meta-analysis. Int. J. Cardiol. 2017, 226, 110-117. [CrossRef]

21. Vaneckova, P.; Bambrick, H. Cause-specific hospital admissions on hot days in Sydney, Australia. PLoS ONE 2013, 8, e55459. [CrossRef]

22. Wilson, L.A.; Morgan, G.G.; Hanigan, I.V.; Johnston, F.H.; Hisham, A.R.; Broome, R.; Gaskin, C.; Jalaludin, B. The impact of heat on mortality and morbidity in the Greater Metropolitan Sydney Region: A case crossover analysis. Environ. Health 2013, 12, 98. [CrossRef]

23. Zanobetti, A.; Peters, A. Disentangling interactions between atmospheric pollution and weather. J. Epidemiol. Community Health 2015, 69, 613-615. [CrossRef]

24. Gordon, C. Role of environmental stress in the physiological response to chemical toxicants. Environ. Res. 2003, 92, 1-7. [CrossRef]

25. Qian, Z.; He, Q.; Lin, H.M.; Kong, L.; Bentley, C.M.; Liu, W.; Zhou, D. High temperatures enhanced acute mortality effects of ambient particle pollution in the 'oven' city of Wuhan, China. Environ. Health Perspect. 2008, 116, 1172-1178. [CrossRef]

26. Stafoggia, M.; Schwartz, J.; Forastiere, F.; Perucci, C.A.; The SISTI Group. Does temperature modify the association between air pollution and mortality? A multicity case-crossover analysis in Italy. Am. J. Epidemiol. 2008, 167, 1476-1485. [CrossRef]

27. Li, G.; Zhou, M.; Cai, Y.; Zhang, Y.; Pan, X. Does temperature enhance acute mortality effects of ambient particle pollution in Tianjin City, China. Sci. Total Environ. 2011, 409, 1811-1817. [CrossRef]

28. Cheng, Y.; Kan, H. Effect of the interaction between outdoor air pollution and extreme temperature on daily mortality in Shanghai, China. J. Epidemiol. 2012, 22, 28-36. [CrossRef]

29. Meng, X.; Zhang, Y.; Zhao, Z.; Duan, X.; Xu, X.; Kan, H. Temperature modifies the acute effect of particulate air pollution on mortality in eight Chinese cities. Sci. Total Environ. 2012, 435-436, 215-221. [CrossRef] 
30. Burkart, K.; Canário, P.; Breitner, S.; Schneider, A.; Scherber, K.; Andrade, H.; Alcoforado, M.J.; Endlicher, W. Interactive short-term effects of equivalent temperature and air pollution on human mortality in Berlin and Lisbon. Environ. Pollut. 2013, 183, 54-63. [CrossRef]

31. Analitis, A.; Michelozzi, P.; D'Ippoliti, D.; de'Donato, F.; Menne, B.; Matthies, F.; Atkinson, R.W.; Iñiguez, C.; Basagaña, X.; Schneider, A.; et al. Effects of heat waves on mortality: Effect modification and confounding by air pollutants. Epidemiology 2014, 25, 15-22. [CrossRef]

32. Breitner, S.; Wolf, K.; Devlin, R.B.; Diaz-Sanchez, D.; Peters, A.; Schneider, A. Short-term effects of air temperature on mortality and effect modification by air pollution in three cities of Bavaria, Germany: A time-series analysis. Sci. Total Environ. 2014, 485-486, 49-61. [CrossRef]

33. Li, L.; Yang, J.; Guo, C.; Chen, P.Y.; Ou, C.Q.; Guo, Y. Particulate matter modifies the magnitude and time course of the non-linear temperature-mortality association. Environ. Pollut. 2015, 196, 423-430. [CrossRef]

34. Hales, S.; Salmond, C.; Town, G.I.; Kjellstron, T.; Woodward, A. Daily mortality in relation to weather and air pollution in Christchurch, New Zealand. Aust. N. Z. J. Public Health 2000, 24, 89-91. [CrossRef]

35. Basu, R.; Feng, W.Y.; Ostro, B. Characterizing temperature and mortality in Nine California Counties. Epidemiology 2008, 19, 138-145. [CrossRef]

36. Ren, C.; Williams, G.M.; Tong, S. Does particulate matter modify the association between temperature and cardiorespiratory diseases? Environ. Health Perspect. 2006, 114, 1690-1696. [CrossRef]

37. IPCC. Managing the Risks of Extreme Events and Disasters to Advance Climate Change Adaptation. In A Special Report of Working Groups I and II of the Intergovernmental Panel on Climate Change; Field, C.B., Barros, V., Stocker, T.F., Qin, D., Dokken, D.J., Ebi, K.L., Mastrandrea, M.D., Mach, K.J., Plattner, G.-K., Allen, S.K., et al., Eds.; Cambridge University Press: Cambridge, UK; New York, NY, USA, 2012; p. 582.

38. Cowan, T.; Purich, A.; Perkins, S.; Pezza, A.; Boschat, G.; Sadler, K. More frequent, longer and hotter heat waves for Australia in the Twenty-First Century. J. Clim. 2014, 27, 5851-5871. [CrossRef]

39. Alexander, L.; Tebaldi, C. Climate and Weather Extremes: Observations, Modelling, And Projections. In The Future of the World's Climate; Henderson-Sellers, A., McGuffie, K., Eds.; Elsevier: New York, NY, USA, 2012; pp. 253-288.

40. Tong, S.; Ren, C.; Becker, N. Excess deaths during the 2004 heat wave in Brisbane, Australia. Int. J. Biometeorol. 2010, 54, 393-400. [CrossRef]

41. Kent, S.T.; McClure, L.A.; Zaitchik, B.F.; Smith, T.T.; Gohlke, J.M. Heat waves and health outcomes in Alabama (USA): The importance of heat wave definition. Environ. Health Perspect. 2014, 122, 151-158. [CrossRef]

42. NSW Office of Environment and Heritage. 2015b. Quality assurance for the air quality monitoring network. Available online: http://www.environment.nsw.gov.au/topics/air/understanding-air-quality-data/ data-validation (accessed on 19 April 2018).

43. Junger, W.L.; Ponce de Leon, A. Imputation of missing data in time series for air pollutants. Atmos. Environ. 2015, 102, 96-104. [CrossRef]

44. Khalaj, B.; Lloyd, G.; Sheppeard, V.; Dear, K. The health impacts of heat waves in five regions of New South Wales, Australia: A case-only analysis. Int. Arch. Occup. Environ. Health 2010, 83, 833-842. [CrossRef]

45. Maclure, M. The case-crossover design: A method for studying transient e ffects on the risk of acute events. Am. J. Epidemiol. 1991, 133, 144-153. [CrossRef]

46. Janes, H.; Sheppard, L.; Lumley, T. Case-crossover analyses of air pollution exposure data: Referent selection strategies and their implications for bias. Epidemiology 2005, 16, 717-726. [CrossRef]

47. Zhang, Y.; Nitschke, M.; Bi, P. Risk factors for direct heat-related hospitalization during the 2009 Adelaide heat wave: A case-crossover study. Sci. Total Environ. 2013, 442, 1-5. [CrossRef]

48. Gronlund, C.J.; Zanobetti, A.; Schwartz, J.D.; Wellenius, G.A.; O'Neill, M.S. Heat, heat waves, and hospital admissions among the elderly in the United States, 1992-2006. Environ. Health Perspect. 2014, 122, 1187-1192. [CrossRef]

49. Tong, S.; Wang, X.Y.; Guo, Y. Assessing the short-term effects of heatwaves on mortality and morbidity in Brisbane, Australia: Comparison of case-crossover and time series analyses. PLoS ONE 2012, 7, e37500. [CrossRef]

50. Bell, M.L.; O’Neill, M.S.; Ranjit, N.; Borja-Aburto, V.H.; Cifuentes, L.A.; Gouveia, N.C. Vulnerability to heat-related mortality in Latin America: a case- crossover study in São Paulo, Brazil, Santiago, Chile and Mexico City, Mexico. Int. J. Epidemiol. 2008, 37, 796-804. [CrossRef]

51. Barnett, A.G.; Dobson, A.J. Analysing seasonal health data. Statistics for Biology and Health; Springer: Berlin, Germany, 2010. 
52. Davis, R.E.; McGregor, G.R.; Enfield, K.B. Humidity: A review and primer on atmospheric moisture and human health. Environ. Res. 2016, 144, 106-116. [CrossRef]

53. Kingsley, S.L.; Eliot, M.N.; Gold, J.; Vanderslice, R.R.; Wellenius, G.A. Current and projected heat-related morbidity and mortality in Rhode Island. Environ. Health Perspect. 2016, 124, 460-467. [CrossRef]

54. Ren, C.; Tong, S. Temperature modifies the health effects of particulate matter in Brisbane, Australia. Int. J. Biometerol. 2006, 51, 87-96. [CrossRef]

55. Qiu, H.; Yu, I.T.; Wang, X.; Tian, L.; Ah Tse, L.; Wai Wong, T. Cool and dry weather enhances the effects of air pollution on emergency IHD hospital admissions. Int. J. Cardiol. 2013, 168, 500-505. [CrossRef]

56. Karagulian, F.; Belis, C.A.; Dora, C.F.C.; Prüss-Ustün, A.M.; Bonjour, S.; Adair-Rohani, H.; Amann, M. Contributions to cities' ambient particulate matter (PM): A systematic review of local source contributions at the global level. Atmos. Environ. 2015, 120, 475-483. [CrossRef]

57. World Health Organization. Concentration of particulate matter with an aerodynamic diameter of $10 \mathrm{um}$ or less (PM10) in nearly 3000 urban areas, 2016, 2008-2015. Available online: http://gamapserver.who.int/ mapLibrary/Files/Maps/Global_pm10_cities.png?ua=1 (accessed on 2 December 2017).

58. Guo, Y.; Gasparrini, A.; Armstrong, B.; Li, S.; Tawatsupa, B.; Tobias, A.; Lavigne, E.; de Sousa Zanotti Stagliorio Coelho, M.; Tobias, A.; Lavigne, E.; et al. Global variation in the effects of ambient temperature on mortality: A systematic evaluation. Epidemiology 2014, 25, 781-789. [CrossRef]

59. Guo, Y.; Gasparrini, A.; Armstrong, B.G.; Tawatsupa, B.; Tobias, A.; Lavigne, E.; de Sousa Zanotti Stagliorio Coelho, M.; Pan, X.; Kim, H.; Hashizume, M. Heat wave and mortality: A multicountry, multicommunity study. Environ. Health Perspect. 2017, 125. [CrossRef]

60. Gordon, C.J.; Mohler, F.S.; Watkinson, W.P.; Rezvani, A.H. Temperature regulation in laboratory mammals following acute toxic insult. Toxicology 1988, 53, 161-178. [CrossRef]

61. Crandall, C.G.; González-Alonso, J. Cardiovascular function in the heat-stressed human. Acta Physiol. 2010, 199, 407-423. [CrossRef]

62. Madaniyazi, L.; Zhou, Y.; Li, S.; Williams, G.; Jaakkola, J.J.K.; Liang, X.; Liu, Y.; Wu, S.; Guo, Y. Outdoor temperature, heart rate, and blood pressure in Chinese adults: Effect modification by individual characteristics. Sci. Rep. 2016, 6, 21003. [CrossRef]

63. Barnett, A.G.; Sans, S.; Salomaa, V.; Kuulasmaa, K.; Dobson, A.J.; WHO MONICA Project. The effect of temperature on systolic blood pressure. Blood Press Monit. 2007, 12, 195-203. [CrossRef]

64. Ren, C.; O'Neill, M.S.; Park, S.K.; Sparrow, D.; Vokonas, P.; Schwartz, J. Ambient temperature, air pollution, and heart rate variability in an aging population. Am. J. Epidemiol. 2011, 173, 1013-1021. [CrossRef]

65. Nelin, T.D.; Joseph, A.M.; Gorr, M.W.; Wold, L.E. Direct and indirect effects of particulate matter on the cardiovascular system. Toxicol. Lett. 2012, 208, 293-299. [CrossRef]

66. Kenney, W.L.; Munce, T.A. Aging and human temperature regulation. J. Appl. Physiol. 2003, 95, $2598-2603$. [CrossRef]

67. Sacks, J.D.; Stanek, L.W.; Luben, T.J.; Johns, D.O.; Buckley, B.J.; Brown, J.S.; Ross, M. Particulate matter induced health effects: Who is susceptible? Environ. Health Perspect. 2011, 119, 446-454. [CrossRef]

68. NSW Health. Physical Activity in Adults. HealthStats NSW. 2017. Available online: http://www.healthstats. nsw.gov.au/Indicator/beh_phys_age/beh_phys_age_snap (accessed on 2 February 2018).

69. Tong, S.; Wang, X.Y.; Barnett, A.G. Assessment of heat-related health impacts in Brisbane, Australia: Comparison of different heat wave definitions. PLoS ONE 2010, 5, e12155. [CrossRef]

70. Anderson, G.B.; Bell, M.L. Weather-related mortality: How heat, cold and heat waves affect mortality in the United States. Epidemiology 2009, 20, 205-213. [CrossRef]

71. D'Ippoliti, D.; Michelozzi, P.; Marino, C.; de'Donato, F.; Menne, B.; Katsouyanni, K.; Kirchmayer, U.; Analitis, A.; Medina-Ramón, M.; Paldy, A.; et al. The impact of heat waves on mortality in 9 European cities: results from the EuroHEAT project. Environ. Health 2010, 9, 37. [CrossRef]

72. Kovats, S.; Hajat, S.; Wilkinson, P. Contrasting patterns of mortality and hospital admissions during hot weather and heat waves in Greater London, UK. Occup. Environ. Med. 2004, 61, 893-898. [CrossRef]

(C) 2019 by the authors. Licensee MDPI, Basel, Switzerland. This article is an open access article distributed under the terms and conditions of the Creative Commons Attribution (CC BY) license (http://creativecommons.org/licenses/by/4.0/). 